\title{
SARS-CoV-2 501Y.V2 escapes neutralization by South African COVID-19 donor plasma
}

\author{
Constantinos Kurt Wibmer', Frances Ayres', Tandile Hermanus ${ }^{1}$, Mashudu Madzivhandila', \\ Prudence Kgagudi', Brent Oosthuysen', Bronwen E. Lambson ${ }^{1,2}$, Tulio de Oliveira3, \\ Marion Vermeulen ${ }^{4}$, Karin van der Berg ${ }^{4,5}$, Theresa Rossouw ${ }^{6}$, Michael Boswell ${ }^{7}$, \\ Veronica Ueckermann7, Susan Meiring ${ }^{1}{ }^{1}$, Anne von Gottberg ${ }^{1,8}$, Cheryl Cohen ${ }^{1,9}$, Lynn Morris (1) 1,2, \\ Jinal N. Bhiman $\mathbb{1}^{1,10,11}$ and Penny L. Moore $\mathbb{1}^{1,2,11 \bowtie}$
}

\begin{abstract}
SARS-CoV-2 501Y.V2 (B.1.351), a novel lineage of coronavirus causing COVID-19, contains substitutions in two immunodominant domains of the spike protein. Here, we show that pseudovirus expressing 501Y.V2 spike protein completely escapes three classes of therapeutically relevant antibodies. This pseudovirus also exhibits substantial to complete escape from neutralization, but not binding, by convalescent plasma. These data highlight the prospect of reinfection with antigenically distinct variants and foreshadows reduced efficacy of spike-based vaccines.
\end{abstract}

Individuals infected with severe acute respiratory syndrome coronavirus 2 (SARS-CoV-2), the virus that causes coronavirus disease 2019 (COVID-19), develop neutralizing antibodies that can persist for months ${ }^{1,2}$. Neutralizing antibodies are considered the primary correlate of protection from infection and are being pursued as therapeutics ${ }^{3,4}$. Interim analyses with monoclonal neutralizing antibodies have shown success, facilitating their authorization for emergency use ${ }^{5,6}$.

The SARS-CoV-2 receptor binding domain (RBD) exists in either an 'up' (receptor-accessible) or 'down' (receptor-shielded) conformation. RBD is the dominant neutralization target for this and other human coronaviruses ${ }^{7,8}$. These antibodies can be broadly divided into four main classes, of which two overlap with the angiotensin converting enzyme 2 (ACE2) receptor binding site (Fig. 1a and Supplementary Fig. 1a) ${ }^{9}$. Class 1 antibodies are most frequently elicited in SARS-CoV-2 infection and include a public antibody response to an epitope only accessible in the RBD 'up' conformation $^{10}$. Class 2 antibodies use more diverse $\mathrm{VH}$-genes and bind to RBD 'up' and RBD 'down' conformations of spike. After RBD, the $\mathrm{N}$-terminal domain (NTD) of spike is the next most frequently targeted by neutralizing antibodies, most of which target a single immunodominant site ${ }^{11}$.

We, and others, recently described a new SARS-CoV-2 lineage in South Africa, defined as Nextstrain clade 20H/501Y.V2 (PANGOLin lineage B.1.351) $)^{12}$. This lineage is defined by nine changes in the spike protein (Supplementary Fig. 1b) relative to the Wuhan-1 D614G spike mutant that previously dominated in South Africa (here referred to as the original lineage) ${ }^{13}$. These changes include N501Y, which confers enhanced affinity for ACE2 (ref. ${ }^{14}$ ) and clusters of substitutions in two immunodominant regions of spike, suggesting escape from neutralization. Indeed, substitutions at $\mathrm{E} 484$ reduce neutralization sensitivity to convalescent plasma ${ }^{15}$. We therefore compared neutralization by monoclonal antibodies and convalescent plasma of 501Y.V2 to Wuhan-1 D614G, using a spike-pseudotyped lentivirus neutralization assay.

An analysis of 17 class I antibody structures revealed their epitopes to be centered on spike residue K417, one of three substitutions in the RBD of the 501Y.V2 lineage. These antibodies contact $60-100 \%$ of residue K417 side-chain-accessible surface area, including key hydrogen bonds at this site (Fig. 1b). Three representative antibodies were assessed by ELISA and achieved saturated binding to recombinant RBD from the original lineage but not 501Y.V2 RBD (Fig. 1c). Similarly, all three antibodies potently neutralized the original lineage, but not the 501Y.V2 pseudovirus (at $25 \mu \mathrm{g} \mathrm{ml}^{-1}$ ), confirming dependence on the K417 residue (Fig. 1d).

A structural analysis of 15 class 2 antibodies and 6 nanobodies revealed key interactions with spike residue E484 (Fig. 1e). Each contacted $40-100 \%$ of the E484 side-chain-accessible surface area and formed critical hydrogen bonds or charged interactions at this site. As with class 1 antibodies, three representative class 2 antibodies failed to bind 501Y.V2 RBD (Fig. 1f) and were unable to neutralize the 501Y.V2 pseudovirus (Fig. 1g). Thus, the SARS-CoV-2 501Y. V2 lineage has effectively escaped two major classes of neutralizing antibodies targeting an immunodominant, highly antigenic site in the RBD of the spike protein.

501Y.V2 is also defined by several changes in the NTD, including a three-amino-acid deletion preceding the N5-loop supersite (Fig. $1 \mathrm{~h}$ and Supplementary Fig. 1b). An analysis of NTD-bound antibody structures showed that roughly half their neutralization interfaces with spike comprised the N5-loop supersite, often involving

'National Institute for Communicable Diseases (NICD) of the National Health Laboratory Service (NHLS), Johannesburg, South Africa. ${ }^{2}$ Antibody Immunity Research Unit, School of Pathology, Faculty of Health Sciences, University of the Witwatersrand, Johannesburg, South Africa. ${ }^{3} \mathrm{Kw}$ wZulu-Natal Research Innovation and Sequencing Platform (KRISP), Department of Laboratory Medicine \& Medical Sciences, University of KwaZulu-Natal, Durban, South Africa, Durban, South Africa. ${ }^{4}$ South African National Blood Service, Weltevreden Park, South Africa. ${ }^{5}$ Division of Clinical Haematology, Faculty of Health Sciences, University of Cape Town, Cape Town, South Africa. ${ }^{6}$ Department of Immunology, Faculty of Health Sciences, University of Pretoria, Pretoria, South Africa. ${ }^{7}$ Division for Infectious Diseases, Department of Internal Medicine, Steve Biko Academic Hospital and University of Pretoria, Pretoria, South Africa. ${ }^{8}$ Department of Clinical Microbiology and Infectious Diseases, Faculty of Health Sciences, University of the Witwatersrand, Johannesburg, South Africa. ' School of Public Health, Faculty of Health Sciences, University of the Witwatersrand, Johannesburg, South Africa. ${ }^{10}$ Department of Virology, Faculty of Health Sciences, University of the Witwatersrand, Johannesburg, South Africa. "'These authors contributed equally: Jinal N. Bhiman, Penny L. Moore. 凶e-mail: pennym@nicd.ac.za 

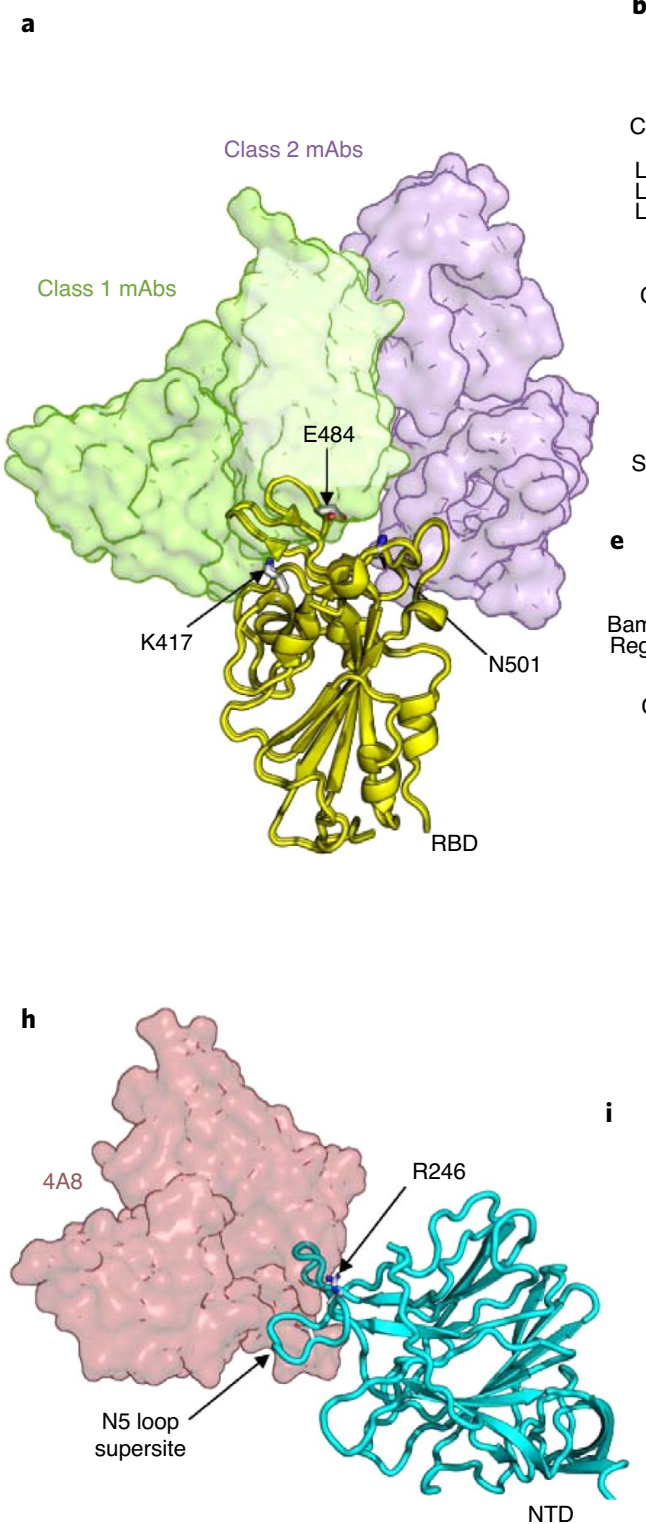

b

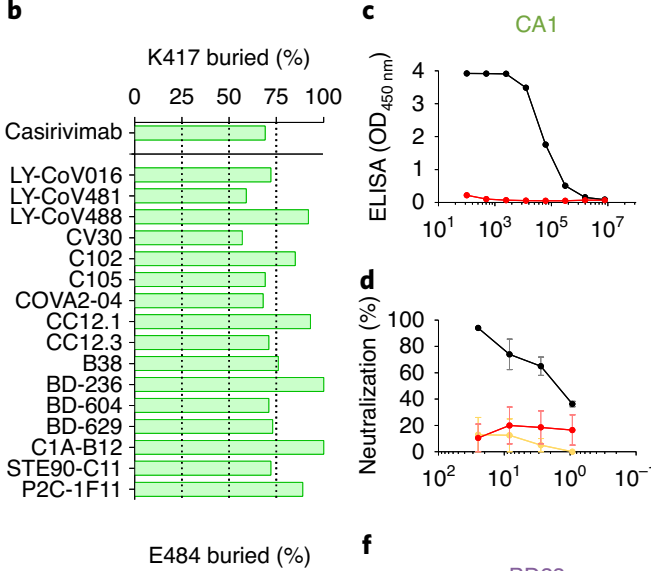

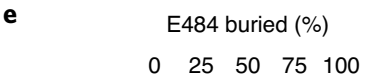
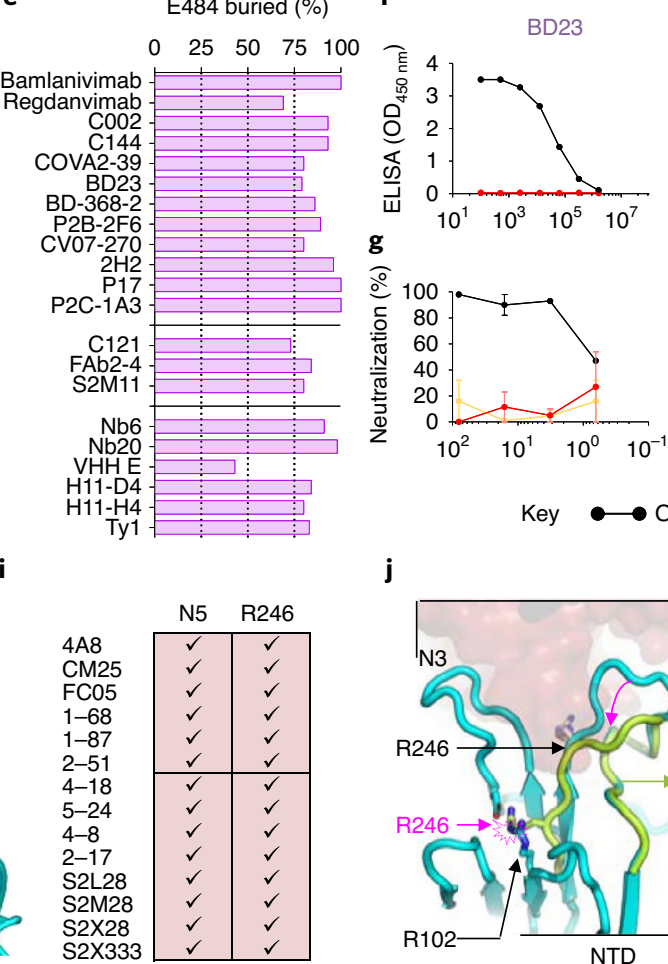

g

Key
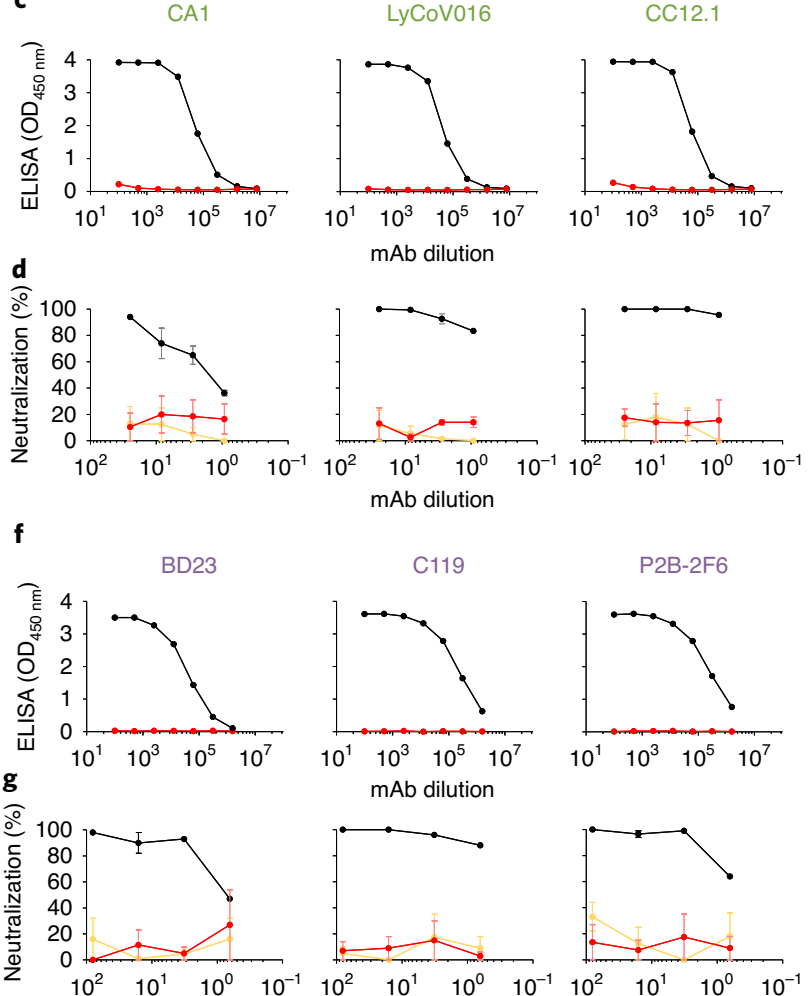

C119

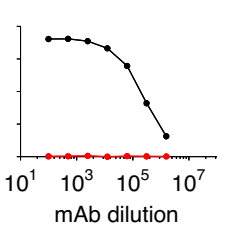

P2B-2F6
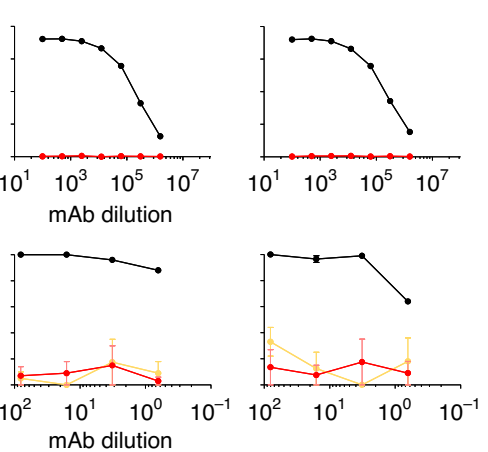

$\bullet$ Original $\longrightarrow$ RBD mutant $\bullet$ 501Y.V2 j

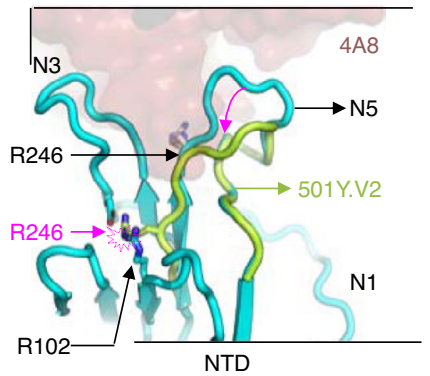

k.

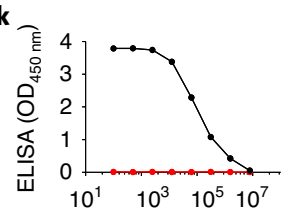

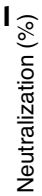

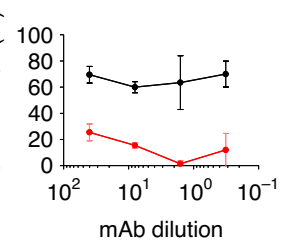

Fig. 1 | SARS-CoV-2 501Y.V2 is resistant to monoclonal antibodies. a, Structure of SARS-CoV-2 RBD (yellow) modeled in complex with class 1 (translucent green) or class 2 (translucent purple) neutralizing antibodies. Side chains of residues K417, E484 and N501 are indicated. mAb, monoclonal antibody. b, A plot showing percentage of K417 accessible surface area ( $x$ axis) buried (buried surface area) in class 1 antibody paratopes (listed on the $y$ axis). VH3-53/66 antibodies are separated below the horizontal line. c, ELISA binding for CA1, LyCoV016 and CC12.1 to the original (black) or the 501Y.V2 RBD (red). d, Neutralization curves for the same antibodies shown in c, against the original pseudovirus (black), $501 Y . V 2$ (red) or a chimeric construct that includes only the RBD substitutions K417N, E484K and N501Y (orange). e, Percentage of E484 accessible surface area buried in class 2 antibody paratopes (listed on y axis). VH1-2 antibodies (middle) or sy-/nanobodies (bottom) are separated with horizontal lines. f, ELISA binding for BD23, C119 and P2B-2F6 to the original (black) or 501Y.V2 RBD (red). g, Neutralization curves for the same antibodies shown in $\mathbf{f}$, against original (black), 501Y.V2 (red) or RBD chimeric pseudoviruses (orange). h, Structure of SARS-CoV-2 NTD (cyan) modeled in complex with VH1-24 neutralizing antibody (translucent maroon). The N5-loop supersite and residue R246 are indicated. i, Contribution of N5 loop and R246 to NTD-directed neutralizing antibodies is indicated. $\mathbf{j}$, Modeling of the $\Delta 242-244$ deletion (lime green). NTD loops N1, N3 and N5 are shown and the position of R246 in the original NTD and 501Y.V2 NTD is labeled with black and pink, respectively. The minimum displacement for 501Y.V2 loop N5 and the accompanying clash with R102 are indicated with pink arrows. k, ELISA binding for 4A8 to original (black) or $501 Y . V 2$ NTD (red). I, Neutralization curves for 4A8 against the original (black) or $501 Y . V 2$ (red) pseudovirus. All experiments were performed in duplicate.

key residue R246 (Fig. 1i). Modeling the 501Y.V2 N5-loop deletion onto the antibody $4 \mathrm{~A} 8$-bound spike structure revealed an apical loop displacement of at least $8 \AA$ away from the $4 \mathrm{~A} 8$ paratope (Fig. 1j). In addition, the deletion would shift position R246 three amino acids earlier, bringing it into proximity with R102 and creating a potential clash that could locally disrupt the N5-loop conformation and shift it even further from the $4 \mathrm{~A} 8$ paratope. Consequently, when assessed by ELISA, 4A8 was unable to bind to recombinant 501Y.V2 NTD (Fig. 1k) and failed to neutralize the 501Y.V2 pseudovirus (Fig. 11), showing escape from N5-loop targeted neutralizing antibodies.

We next sought to evaluate the effect of 501Y.V2 spike substitutions on polyclonal plasma/sera derived from individuals with 


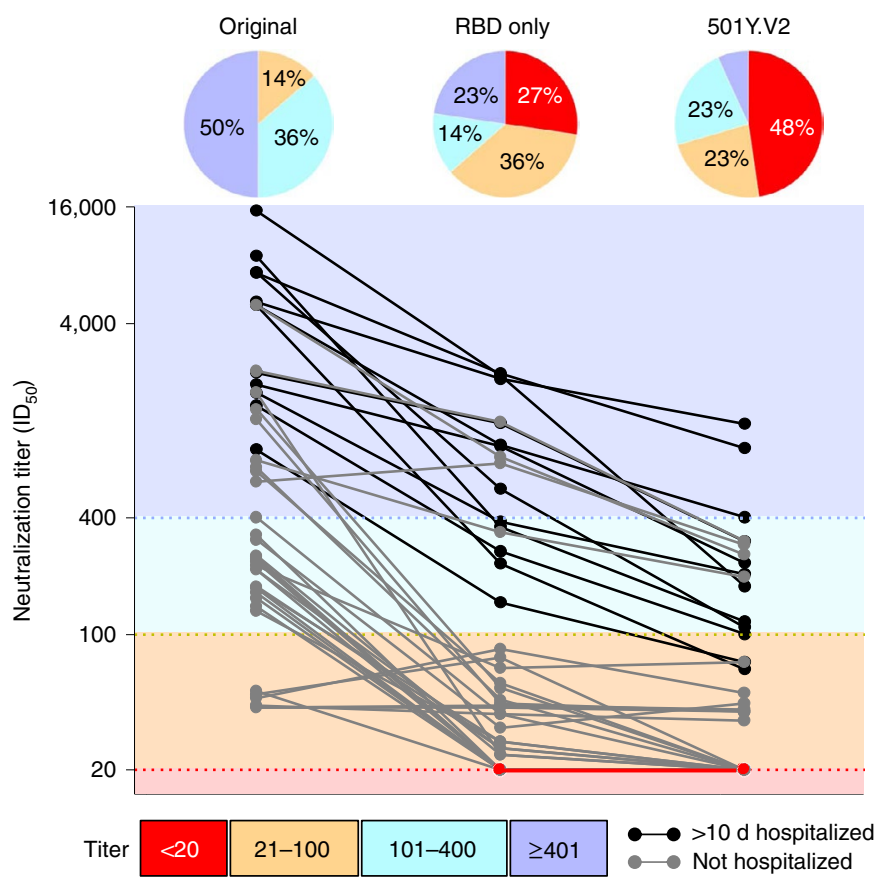

Fig. 2 | SARS-CoV-2 501Y.V2 increased resistance to neutralization by convalescent plasma/serum. Plasma/serum collected from individuals infected with SARS-CoV-2 was assessed for neutralization to the original lineage (Wuhan-1 D614G, left), an RBD chimeric mutant containing K417N, E484K and N501Y substitutions only (middle) or the 501Y.V2 lineage pseudovirus. Twelve of the samples were collected from donors hospitalized for $>10 \mathrm{~d}$ with COVID-19 (black). The graph is colored according to the magnitude of neutralization titer, with $I D_{50}$ greater or lesser than 1:400 colored dark or light blue, respectively and titer $<100$ colored orange. The limit of detection (knockout) was an $\mathrm{ID}_{50}<20$ (red). Pie charts above each set of data points summarize the proportion of samples in each titer group.

PCR-confirmed SARS-CoV-2 infection, including individuals who were hospitalized with severe COVID-19. Samples were divided into two groups, half with higher titer neutralizing antibodies (22 of $44,50 \%$ inhibitory dilution $\left(\mathrm{ID}_{50}\right)>400$ ) and half with lower titers (22 of 44, 400 $\geq \mathrm{ID}_{50}>25$ ) to the original SARS-CoV-2 D614G lineage (Fig. 2 and Supplementary Fig. 2a). Consistent with previous studies, when stratified by disease severity, convalescent individuals who reported mild-to-moderate disease developed substantially lower neutralizing antibody titers (average $\mathrm{ID}_{50}$ titer $488, n=30$ ) than severely ill individuals from the hospitalized cohorts (average $\mathrm{ID}_{50}$ titer $4,212, n=14$ ).

When these same samples were assessed against the 501Y.V2 pseudovirus, nearly half ( 21 of $44,48 \%)$ had no detectable neutralization activity (and $71 \%$ had $\mathrm{ID}_{50}<100$ ). Only three samples (7\%) retained titers of $\mathrm{ID}_{50}>400$ (Fig. 2 and Supplementary Fig. 2a). Notably, these three samples were obtained from individuals reporting severe disease and had among the highest neutralization titers against the original virus. Conversely, four samples with borderline neutralization of the original virus were unaffected by $501 \mathrm{Y}$. V2 substitutions, perhaps representing additional, less-potent specificities. To define the location of dominant escape substitutions, neutralization was also assessed against the RBD chimeric pseudovirus containing only three 501Y.V2 substitutions (K417N, E484K and N501Y) (Fig. 2 and Supplementary Fig. 2a). Substantial loss of neutralization was also observed against the RBD-only mutant, with $27 \%$ of the samples losing all activity against the RBD triple mutants $\left(63 \%\right.$ had $\left.\mathrm{ID}_{50}<100\right)$ and only $23 \%$ retaining higher titers of $\mathrm{ID}_{50}>400$. These data provide more evidence for the dominance of class 1 and class 2 neutralizing antibodies in polyclonal sera; however, differences in neutralization between RBD-only chimera and 501Y.V2 also highlight the contribution of 501Y.V2 NTD substitutions (L18F, D80A, D215G and $\Delta 242-244$ ) to neutralization escape. This was particularly evident in higher titer samples, which retained an average $\mathrm{ID}_{50}$ titer of 680 against the RBD-only mutant.

While neutralizing antibodies to SARS-CoV-2 are dominated by the specificities defined above, non-neutralizing antibodies are also elicited during SARS-CoV-2 infection. To determine whether 501Y. $\mathrm{V} 2$ is still recognized by non-neutralizing antibodies, the binding of polyclonal sera (from Fig. 2) to a recombinant protein that includes the RBD and subdomain 1 (RBD+SBD1) of 501Y.V2 or the original lineage was assessed by ELISA (Supplementary Fig. 2b). These data revealed that binding of polyclonal plasma to 501Y.V2 $\mathrm{RBD}+\mathrm{SBD} 1$ was only substantially affected in a minority of cases (14 of 44 with more than a fivefold reduction, 32\%). Most of the convalescent plasma/serum suffered less than a four-fold reduction in total binding activity (as calculated by area under the curve), suggesting a considerable non-neutralizing antibody component is still able to bind the 501Y.V2 spike.

Among previous emerging lineages, only D614G has subsequently become globally dominant ${ }^{16}$. The repeated, independent evolution of spike position 501 in 501Y.V1 (https://virological.org/t/576), 501Y.V2 (ref. ${ }^{12}$ ) and 501Y.V3 https://virological.org/t/586), strongly argues for a selective advantage, likely enhanced transmissibility, of these new variants. Here we have shown that the 501Y.V2 lineage, containing nine spike substitutions, and rapidly emerging in South Africa during the second half of 2020, is resistant to neutralizing antibodies found in $48 \%$ of individuals infected with previously circulating lineages. These data, showing a 13 -fold reduction in mean titer, are corroborated by vesicular stomatitis virus-pseudotyped and live virus assays showing an 11- to 33-fold and 6- to 204-fold reduction in mean titer (including complete knock out) relative to the original lineage, respectively ${ }^{17,18}$. The 501Y.V3 lineage has similar changes including $417 \mathrm{~T}$ and $484 \mathrm{~K}$ (in RBD) as well as $18 \mathrm{~F}$ and $20 \mathrm{~N}$ (in NTD), thus also having strong potential for high levels of neutralization resistance. The independent emergence and subsequent selection of 501Y lineages with key substitutions conferring neutralization resistance strongly argues for selection by neutralizing antibodies as the dominant driver for SARS-CoV-2 spike diversification and makes these lineages of considerable public health concern. This suggests that, despite the many people who have already been infected with SARS-CoV-2 globally and are presumed to have accumulated some level of immunity, new variants such as 501Y.V2 may pose a substantial reinfection risk.

While higher titers of neutralizing antibodies are common in hospitalized individuals, most people infected with SARS-CoV-2 develop low-to-moderate neutralization titers ${ }^{2}$. Therefore, the data herein suggest that most individuals infected with previous SARS-CoV-2 lineages will have greatly reduced neutralization activity against 501Y.V2. This dramatic effect on plasma neutralization can be explained by the dominance of RBD-directed neutralizing antibodies, supported by studies showing reduced plasma neutralization titers mediated by the $\mathrm{E} 484 \mathrm{~K}$ change alone ${ }^{15}$. Notably, here we show that the $\mathrm{K} 417 \mathrm{~N}$ change also has a crucial role in viral escape, effectively abrogating neutralization by a well-defined, multidonor class of VH3-53/66 germline-restricted public antibodies that comprise some of the most common and potent neutralizing antibodies to SARS-CoV-2 $\left(\operatorname{ref}^{8}\right)$.

The marked loss of neutralization against 501Y.V2 pseudovirus compared to the RBD-only chimeric pseudovirus demonstrates the important role that substitutions in the NTD play in mediating immune escape. For 501Y.V2 this resistance to neutralization is likely mediated by a three-amino-acid deletion that completely disrupts a dominant public antibody response to the N5-loop 
supersite $^{11}$. This deletion predominates among 501Y.V2 variants and occurs either alone or with an R246I substitution that is also important for neutralization by several NTD-directed neutralizing antibodies.

The relatively rapid acquisition of a comprehensive suite of neutralization escape substitutions likely occurred because of the large number of commonly shared public antibodies (such as VH3-53/66, VH1-2 and VH1-24) to both the RBD and NTD of spike, together with high levels of SARS-CoV-2 transmission. The sporadic emergence of escape substitutions in long-term viral shedders, including immunocompromised individuals, may also contribute to the emergence of neutralization-resistant viruses ${ }^{19}$. Altogether, these data highlight the need for increased, ongoing genomic surveillance during the SARS-CoV-2 pandemic.

Crucially, it is from these same public antibody responses that many therapeutic strategies currently under development have been derived ${ }^{4}$. The overwhelming majority of monoclonal antibodies already on the path to licensure target residues K417 or E484 and are therefore likely to be futile against 501Y.V2. In addition, emerging variants may limit the use of recently identified neutralizing antibodies that target the NTD N5-loop supersite. Some of these monoclonal antibodies have already been granted emergency use authorization in the United States (Regeneron Pharmaceuticals and Eli Lilly and Company), including antibodies ineffective against 501Y.V2 (such as REGN10933 and LY-CoV555) as well as antibodies likely to retain neutralization of this variant (REGN10987 and VIR-7831), some of which are being engineered to potentially enhance virus-specific T cell function (VIR-7832).

These data also have implications for the effectiveness of SARS-CoV-2 vaccines, largely based on immune responses to the original spike protein. Indeed, sera from the Moderna and Pfizer-BioNTech vaccinees show significantly reduced neutralization of 501Y.V2 (ref. ${ }^{18}$ ). Furthermore, compared to global efficacy estimates, vaccine efficacy in South Africa (overlapping with the emergence of 501Y.V2) has been significantly reduced for several vaccines. Conversely, these same trials seem to retain efficacy against severe COVID-19. While antibody effector functions elicited by infection and vaccination have been implicated in protecting from reinfection and disease $\mathrm{e}^{20}$, the role of non-neutralizing antibodies and the efficacy of $\mathrm{T}$ cell responses to 501Y.V2 remain to be elucidated. We did not measure the extent of these responses to 501Y. V2 but did show that a substantial proportion of non-neutralizing antibodies remain active against 501Y.V2 recombinant RBD protein. Ultimately, the correlates of protection against SARS-CoV-2 infection and severe COVID-19 disease remain undetermined and rely upon ongoing large-scale clinical trials. Nevertheless, these data highlight the urgent requirement for rapidly adaptable vaccine design platforms and the need to identify less-mutable viral targets for incorporation into future immunogens.

\section{Online content}

Any methods, additional references, Nature Research reporting summaries, source data, extended data, supplementary information, acknowledgements, peer review information; details of author contributions and competing interests; and statements of data and code availability are available at https://doi.org/10.1038/ s41591-021-01285-x.

Received: 21 January 2021; Accepted: 10 February 2021;

Published online: 2 March 2021

\section{References}

1. Zhou, P. et al. A pneumonia outbreak associated with a new coronavirus of probable bat origin. Nature 579, 270-273 (2020).

2. Wajnberg, A. et al. Robust neutralizing antibodies to SARS-CoV-2 infection persist for months. Science 370, 1227-1230 (2020).

3. Mercado, N. B. et al. Single-shot Ad26 vaccine protects against SARS-CoV-2 in rhesus macaques. Nature 586, 583-588 (2020).

4. Marovich, M., Mascola, J. R. \& Cohen, M. S. Monoclonal antibodies for prevention and treatment of COVID-19. JAMA 324, 131-132 (2020)

5. Weinreich, D. M. et al. REGN-COV2, a neutralizing antibody cocktail, in outpatients with COVID-19. N. Engl. J. Med. 384, 238-251 (2021).

6. Chen, P. et al. SARS-CoV-2 neutralizing antibody LY-CoV555 in outpatients with COVID-19. N. Engl. J. Med. 384, 229-237 (2021).

7. Piccoli, L. et al. Mapping neutralizing and immunodominant sites on the SARS-CoV-2 spike receptor-binding domain by structure-guided high-resolution serology. Cell 183, 1024-1042 (2020).

8. Barnes, C. O. et al. Structures of human antibodies bound to SARS-CoV-2 spike reveal common epitopes and recurrent features of antibodies. Cell 182, 828-842 (2020)

9. Barnes, C. O. et al. SARS-CoV-2 neutralizing antibody structures inform therapeutic strategies. Nature 588, 682-687 (2020).

10. Yuan, M. et al. Structural basis of a shared antibody response to SARS-CoV-2. Science 369, 1119-1123 (2020).

11. Chi, X. et al. A neutralizing human antibody binds to the N-terminal domain of the spike protein of SARS-CoV-2. Science 369, 650-655 (2020).

12. Tegally, H. et al. Emergence and rapid spread of a new severe acute respiratory syndrome-related coronavirus 2 (SARS-CoV-2) lineage with multiple spike mutations in South Africa. Preprint at medRxiv https://doi. org/10.1101/2020.12.21.20248640 (2020).

13. Tegally, H. et al. Sixteen novel lineages of SARS-CoV-2 in South Africa. Nat. Med. https://doi.org/10.1038/s41591-021-01255-3 (2021).

14. Starr, T. N. et al. Deep mutational scanning of SARS-CoV-2 receptor binding domain reveals constraints on folding and ACE2 binding. Cell 182, 1295-1310 (2020).

15. Weisblum, Y. et al. Escape from neutralizing antibodies by SARS-CoV-2 spike protein variants. eLife 9, e61312 (2020).

16. Korber, B. et al. Tracking changes in SARS-CoV-2 spike: evidence that D614G increases infectivity of the COVID-19 virus. Cell 182, 812-827 (2020)

17. Cele, S. et al. Escape of SARS-CoV-2 501Y.V2 variants from neutralization by convalescent plasma. Preprint at medRxiv https://doi. org/10.1101/2021.01.26.21250224 (2021).

18. Wang, P. et al. Increased resistance of SARS-CoV-2 Variants B.1.351 and B.1.1.7 to antibody neutralization. Preprint at bioRxiv https://doi. org/10.1101/2021.01.25.428137 (2021).

19. Choi, B. et al. Persistence and evolution of SARS-CoV-2 in an immunocompromised host. N. Engl. J. Med. 383, 2291-2293 (2020).

20. Atyeo, C. et al. Distinct early serological signatures track with SARS-CoV-2 survival. Immunity 53, 524-532 (2020).

Publisher's note Springer Nature remains neutral with regard to jurisdictional claims in published maps and institutional affiliations.

(C) The Author(s), under exclusive licence to Springer Nature America, Inc. 2021 


\section{Methods}

Samples and ethics approvals. Plasma/serum samples were obtained from individuals without human immunodeficiency virus (HIV), enrolled into one of three studies described below. All participants provided informed consent.

Hospitalized Steve Biko cohort. This study has been given ethics approval by the University of Pretoria, Human Research Ethics Committee (Medical) (247/2020) Serum samples were obtained (longitudinally) from hospitalized patients with PCR-confirmed SARS-CoV-2 infection, known HIV status and aged $\geq 18$ years. Samples from six participants with symptom onset between May and August 2020 were used.

Novel coronavirus (COVID-19) viral shedding and clinical characterization study. This study has been given ethics approval by the University of the Witwatersrand Human Research Ethics Committee (Medical) M160667. Serum samples were obtained (longitudinally) from hospitalized patients with PCR-confirmed SARS-CoV-2 infection, known HIV status and aged $\geq 18$ years. Samples from six participants with symptom onset between May and September 2020 were used.

South African National Blood Service. Plasma was obtained from blood donors of the South African National Blood Service (ethics clearance from South African National Blood Service Human Research Ethics Committee 2019/0519), who had PCR-confirmed SARS-CoV-2 infection, had recovered and were at least $28 \mathrm{~d}$ post-symptom onset. All donors met the standard eligibility criteria for donors who donate source plasma, which includes being generally healthy, being older than 18 years of age, weighing more than $55 \mathrm{~kg}$ and leading a lifestyle that reduces risk of acquiring transfusion transmissible infections. Only male and nulliparous females were accepted as COVID-19 convalescent plasma donors.

Structural modeling. The closed prefusion SARS-CoV-2 spike protein from Nextstrain ${ }^{21}$ clade 501Y.V2 (PANGOLin lineage B.1.351, https://cov-lineages. org/pangolin.html) was modeled on PDBID 6VXX ${ }^{22}$, while a model of the RBD was averaged using neutralizing domain-bound structures compared to the ACE2-bound PDBID 6LZG ${ }^{23}$. Class 1, 2, 3 and 4 antibodies were represented with Fab domains from CV30 (6XE1), C104 (7k8u), VIR-7831 (6WPS) and EY6A (6ZCZ). The 501Y.V2 NTD domain was modeled using both antibody-constrained PDBID 7C2 $\mathrm{L}^{11}$ and unconstrained structures of spike deposited in the protein databank ${ }^{24}$. Involvement of the N5-loop supersite was estimated from available preprint data ${ }^{25-29}$. Buried interfaces were calculated using PDBePISA (https:// www.ebi.ac.uk/pdbe/pisa/pistart.html) and analyzed according to neutralization class $^{30-33}$. All structures were visualized in $\mathrm{PyMol}^{34}$.

Expression and purification of SARS-CoV-2 antigens. Monoclonal antibodies or recombinant SARS-CoV-2 RBD + SBD1 or NTD proteins were transfected in FreeStyle 293F suspension cells (Life Technologies) using PEIMAX transfection reagent (Polysciences). Transfections were incubated at 220 r.p.m., $37^{\circ} \mathrm{C}, 9 \% \mathrm{CO}_{2}$ for $6-7 \mathrm{~d}$ and clarified supernatants were purified using nickel affinity or protein $\mathrm{A}$ and size-exclusion chromatography.

ELISA. Recombinant RBD + SBD1 proteins were coated at $2 \mu \mathrm{g} \mathrm{ml}^{-1}$ onto 96-well, high-binding plates and incubated overnight at $4{ }^{\circ} \mathrm{C}$. The plates were then washed and incubated at $37^{\circ} \mathrm{C}$ for $1-2 \mathrm{~h}$ in blocking buffer (5\% skimmed milk powder, $0.05 \%$ Tween $20,1 \times$ PBS). Plasma samples were added at 1:100 dilution and serially diluted fivefold in blocking buffer. Following a 1 -h incubation at $37^{\circ} \mathrm{C}$, an anti-human horseradish peroxidase-conjugated antibody was added for $1 \mathrm{~h}$ at $37^{\circ} \mathrm{C}$. The signal was developed with TMB substrate (Thermo Fisher Scientific) for $5 \mathrm{~min}$ at room temperature, followed by addition of $1 \mathrm{M} \mathrm{H}_{2} \mathrm{SO}_{4}$ stop solution. Absorbance at $450 \mathrm{~nm}$ was measured and used to calculate area under the curve using GraphPad Prism v.8.4.2.

Lentiviral pseudovirus production and neutralization assay. The 293T/ACE2. MF cells modified to overexpress human ACE2 were kindly provided by M. Farzan (Scripps Research). Cells were cultured in DMEM (Gibco BRL Life Technologies) containing $10 \%$ heat-inactivated serum (FBS) and $3 \mu \mathrm{g} \mathrm{ml}^{-1}$ puromycin at $37^{\circ} \mathrm{C}$, $5 \% \mathrm{CO}_{2}$. Cell monolayers were disrupted at confluency by treatment with $0.25 \%$ trypsin in $1 \mathrm{mM}$ EDTA (Gibco BRL Life Technologies).

The SARS-CoV-2, Wuhan-1 spike, cloned into pCDNA3.1 was mutated using the QuikChange Lightning Site-Directed Mutagenesis kit (Agilent Technologies) to include D614G (original) or K417N, E484K, N501Y, D614G (RBD only) or L18F, D80A, D215G, $\Delta 242-244$, K417N, E484K, N501Y, D614G, A701V (501Y.V2). Pseudoviruses were produced by co-transfection with a lentiviral backbone (HIV-1 pNL4.luc encoding the firefly luciferase gene) and either of the SARS-CoV-2 spike plasmids with PEIMAX (Polysciences). Culture supernatants were clarified of cells by a $0.45-\mu \mathrm{M}$ filter and stored at $-70{ }^{\circ} \mathrm{C}$.

Plasma/serum samples were heat-inactivated and clarified by centrifugation. Pseudovirus and serially diluted plasma/sera were incubated for $1 \mathrm{~h}$ at $37^{\circ} \mathrm{C}, 5 \%$ $\mathrm{CO}_{2}$. Cells were added at $1 \times 104$ cells per well after $72 \mathrm{~h}$ of incubation at $37^{\circ} \mathrm{C}$, $5 \% \mathrm{CO}_{2}$, luminescence was measured using PerkinElmer Life Sciences Model Victor X luminometer. Neutralization was measured as described by a reduction in luciferase gene expression after single-round infection of 293T/ACE2.MF cells with spike-pseudotyped viruses. Titers were calculated as the reciprocal plasma dilution $\left(\mathrm{ID}_{50}\right)$ causing $50 \%$ reduction of relative light units. Equivalency was established through participation in the SARS-CoV-2 Neutralizing Assay Concordance Survey Concordance Survey 1 run by EQAPOL and VQU, Duke Human Vaccine Institute Cell-based neutralization assays using live virus or pseudovirus have demonstrated high concordance, with highly correlated $50 \%$ neutralization titers (Pearson $r=0.81-0.89)^{35}$

Statistical analyses. A Wilcoxon matched pairs signed-rank test showed significant difference in neutralization $\mathrm{ID}_{50}$ titer between the original pseudovirus and the RBD-only mutant or N501Y.V2, as well as between the RBD-only mutant and the 501Y.V2 lineage $(P \leq 0.0001)$.

Reporting Summary. Further information on research design is available in the Nature Research Reporting Summary linked to this article.

\section{Data availability}

The authors confirm that all relevant data are included in the paper and/or its supplementary files.

\section{References}

21. Hadfield, J. et al. Nextstrain: real-time tracking of pathogen evolution. Bioinformatics 34, 4121-4123 (2018).

22. Walls, A. C. et al. Structure, function, and antigenicity of the SARS-CoV-2 spike glycoprotein. Cell 183, 1735 (2020).

23. Wang, Q. et al. Structural and functional basis of SARS-CoV-2 entry by using human ACE2. Cell 181, 894-904 (2020).

24. Berman, H. M. et al. The protein data bank. Nucleic Acids Res. 28 , 235-242 (2000)

25. McCallum, M. et al. N-terminal domain antigenic mapping reveals a site of vulnerability for SARS-CoV-2. Preprint at bioRxiv https://doi. org/10.1101/2021.01.14.426475 (2021)

26. Cerutti, G. et al. Potent SARS-CoV-2 neutralizing antibodies directed against spike $\mathrm{N}$-terminal domain target a single supersite. Preprint at bioRxiv https:// doi.org/10.1101/2021.01.10.426120 (2021).

27. Voss, W. N. et al. Prevalent, protective, and convergent IgG recognition of SARS-CoV-2 non-RBD spike epitopes in COVID-19 convalescent plasma. Preprint at bioRxiv https://doi.org/10.1101/2020.12.20.423708 (2020).

28. Li, D. et al. The functions of SARS-CoV-2 neutralizing and infection-enhancing antibodies in vitro and in mice and nonhuman primates. Preprint at bioRxiv https://doi.org/10.1101/2020.12.31.424729 (2021).

29. Suryadevara, N. et al. Neutralizing and protective human monoclonal antibodies recognizing the N-terminal domain of the SARS-CoV-2 spike protein. Preprint at bioRxiv https://doi.org/10.1101/2021.01.19.427324 (2021).

30. Rapp, M. et al. Modular basis for potent SARS-CoV-2 neutralization by a prevalent VH1-2-derived antibody class. Preprint at bioRxiv https://doi. org/10.1101/2021.01.11.426218 (2021).

31. Schoof, M. et al. An ultrapotent synthetic nanobody neutralizes SARS-CoV-2 by stabilizing inactive spike. Science 370, 1473-1479 (2020).

32. Xiang, Y. et al. Versatile and multivalent nanobodies efficiently neutralize SARS-CoV-2. Science 370, 1479-1484 (2020)

33. Koenig, P. A. et al. Structure-guided multivalent nanobodies block SARS-CoV-2 infection and suppress mutational escape. Science 12, eabe6230 (2021).

34. The PyMOL Molecular Graphics System, version 1.8 (Schrodinger, LLC, 2015).

35. Sholukh, A. M. et al. Evaluation of SARS-CoV-2 neutralization assays for antibody monitoring in natural infection and vaccine trials. Preprint at medRxiv https://doi.org/10.1101/2020.12.07.20245431 (2020).

\section{Acknowledgements}

We thank the COVID-19 convalescent plasma donors, the staff of the South African National Blood Services for contributing samples that enabled this work, Z. van der Walt, T. de Villiers, W. van Hougenhouck-Tulleken for contributing to patient management and sample collection and A. Buys for technical support. We thank the participants of the Novel Coronavirus (COVID-19) Viral Shedding and Clinical Characterization Study (University of the Witwatersrand Health Research Ethics (Medical) committee reference M160667) who contributed samples and the GERMS-SA clinical staff for their contributions to sample and data collection. We acknowledge funding from the South African Medical Research Council (ref. nos. 96825, SHIPNCD 76756 and DST/CON 0250/2012), the Wellcome Trust (grant no. 221003/Z/20/Z), US Centers for Disease Control and Prevention (grant no. 5 U01IP001048-05-00) and the ELMA South Africa Foundation (grant no. 20-ESA011). P.L.M. is supported by the South African Research Chairs Initiative of the Department of Science and Innovation and the National Research Foundation of South Africa (grant no. 98341). C.K.W. is supported by Fogarty International Center of the National Institutes of Health under Award Number R21TW011454 (this work is solely the responsibility of the authors and does not 
necessarily represent the official views of the National Institutes of Health) as well as the FLAIR Fellowship Program under award number FLR\R1\201782 (the FLAIR Fellowship Program is a partnership between the African Academy of Sciences and the Royal Society funded by the UK Government's Global Challenges Research Fund). K.v.d.B. is supported in part by the Fogarty International Centre or the National Institutes of Health under award no. 1D43TW010345. We thank N. Doria-Rose, D. Montefiori, E. Landais and M. Farzan for reagents and assistance in establishing the SARS-CoV-2 pseudotyped neutralization assay and enabling equivalency and proficiency testing. We thank D. Sok, E. Landais, D. Burton, N. Doria-Rose and P. Kwong for SARS-Co-V2-directed monoclonal antibodies. We are grateful to T. Moyo-Gwete and Z. Molaudzi for expressing monoclonal antibodies. We thank the informal 501Y.V2 consortium of South African scientists, chaired by W. Hanekom and T. D'Oliveira for suggestions and discussion of data. We also thank all NGS-SA laboratories in South Africa that were responsible for producing the SARS-CoV-2 genomes that enabled the rapid dissemination of SARS-CoV-2 sequences and the identification of 501Y.V2.

\section{Author contributions}

C.K.W., J.N.B. and P.L.M. conceived the study, designed experiments, analyzed data and wrote the paper. C.K.W., F.A., B.O. and B.E.L. made molecular constructs and expressed antibodies. C.K.W. expressed and purified recombinant antigens. C.K.W.,
T.H., M.M. and P.K. made pseudoviruses, while T.H., M.M. and P.K. performed and analyzed neutralization experiments. J.N.B. and T.d.O. contributed to the identification of the 501Y.V2 lineage and the substitutions comprised therein. L.M. contributed to establishing SARS-CoV-2 assays and infrastructure. M.V., K.v.d.B., T.R., M.B., V.U., S.M., A.v.G. and C.H. provided sera from convalescent donors and associated clinical information. All authors critically reviewed and approved the final manuscript.

\section{Competing interests}

The authors declare no competing interests.

\section{Additional information}

Supplementary information The online version contains supplementary material available at https://doi.org/10.1038/s41591-021-01285-x.

Correspondence and requests for materials should be addressed to P.L.M.

Peer review information Nature Medicine thanks Rogier Sanders and the other, anonymous, reviewers for their contribution to the peer review of this work. Alison Farrell is the primary editor on this article and managed its editorial process and peer review in collaboration with the rest of the editorial team.

Reprints and permissions information is available at www.nature.com/reprints. 


\section{Reporting Summary}

Nature Research wishes to improve the reproducibility of the work that we publish. This form provides structure for consistency and transparency in reporting. For further information on Nature Research policies, see our Editorial Policies and the Editorial Policy Checklist.

\section{Statistics}

For all statistical analyses, confirm that the following items are present in the figure legend, table legend, main text, or Methods section.

$\mathrm{n} / \mathrm{a}$ Confirmed

$\bigotimes$ The exact sample size $(n)$ for each experimental group/condition, given as a discrete number and unit of measurement

$\bigotimes$ A statement on whether measurements were taken from distinct samples or whether the same sample was measured repeatedly

$\varnothing$ The statistical test(s) used AND whether they are one- or two-sided

Only common tests should be described solely by name; describe more complex techniques in the Methods section.

Х $\square$ A description of all covariates tested

Х $\square$ A description of any assumptions or corrections, such as tests of normality and adjustment for multiple comparisons

Q A full description of the statistical parameters including central tendency (e.g. means) or other basic estimates (e.g. regression coefficient) AND variation (e.g. standard deviation) or associated estimates of uncertainty (e.g. confidence intervals)

For null hypothesis testing, the test statistic (e.g. $F, t, r$ ) with confidence intervals, effect sizes, degrees of freedom and $P$ value noted Give $P$ values as exact values whenever suitable.

Х $\square$ For Bayesian analysis, information on the choice of priors and Markov chain Monte Carlo settings

Х $\square$ For hierarchical and complex designs, identification of the appropriate level for tests and full reporting of outcomes

$\triangle \square$ Estimates of effect sizes (e.g. Cohen's d, Pearson's $r$ ), indicating how they were calculated

Our web collection on statistics for biologists contains articles on many of the points above.

\section{Software and code}

Policy information about availability of computer code

Data collection n/a

For manuscripts utilizing custom algorithms or software that are central to the research but not yet described in published literature, software must be made available to editors and

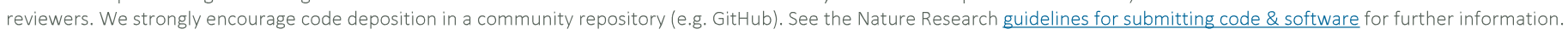

\section{Data}

Policy information about availability of data

All manuscripts must include a data availability statement. This statement should provide the following information, where applicable:

- Accession codes, unique identifiers, or web links for publicly available datasets

- A list of figures that have associated raw data

- A description of any restrictions on data availability

Authors can confirm that all relevant data are included in the paper and/or its supplementary information files. 
Please select the one below that is the best fit for your research. If you are not sure, read the appropriate sections before making your selection.

\ Life sciences

Behavioural \& social sciences

Ecological, evolutionary \& environmental sciences

For a reference copy of the document with all sections, see nature.com/documents/nr-reporting-summary-flat.pdf

\section{Life sciences study design}

All studies must disclose on these points even when the disclosure is negative.
Sample size
44 Plasma donors
Data exclusions
n/a
Replication
Samples were tested in duplicate in neutralization assays and ELISAs. Each assay was repeated twice.
Randomization
$n / a$
Blinding
$n / a$

\section{Reporting for specific materials, systems and methods}

We require information from authors about some types of materials, experimental systems and methods used in many studies. Here, indicate whether each material, system or method listed is relevant to your study. If you are not sure if a list item applies to your research, read the appropriate section before selecting a response.

\begin{tabular}{l|l} 
Materials \& experimental system \\
\hline$n / a$ & Involved in the study \\
\hline & $\bigotimes$ Antibodies \\
$\square$ & $\bigotimes$ Eukaryotic cell lines \\
$\searrow$ & $\square$ Palaeontology and archaeology \\
$\square$ & $\square$ Humals and other organisms \\
$\square$ & $\bigotimes$ Clinical data \\
$\searrow$ & $\square$ Dual use research of concern
\end{tabular}

Methods

$\mathrm{n} / \mathrm{a}$ Involved in the study

Х $\square$ chip-seq

Х $\square$ Flow cytometry

Х $\square$ MRI-based neuroimaging

\section{Antibodies}

$\begin{array}{ll}\text { Antibodies used } & \text { CA1, Ly-CoV016, CC12.1, BD23, C119, P2B-2F6, 4A8, CC12.23, Palivizumab } \\ \text { Validation } & \text { Antibodies were cloned, expressed and quality controlled according to published IC50 data. }\end{array}$

\section{Eukaryotic cell lines}

Policy information about cell lines

Cell line source(s)

Mike Farzan, The Scripps Research Institute

Authentication

Flow cytometry was used to confirm increased ACE-2 expression as reported

Mycoplasma contamination

Commonly misidentified lines

(See ICLAC register) 
Policy information about clinical studies

All manuscripts should comply with the ICMJE guidelines for publication of clinical research and a completed CONSORT checklist must be included with all submissions.

Clinical trial registration Provide the trial registration number from ClinicalTrials.gov or an equivalent agency.

Study protocol

Note where the full trial protocol can be accessed $O R$ if not available, explain why.

Data collection

Describe the settings and locales of data collection, noting the time periods of recruitment and data collection.

Outcomes

Describe how you pre-defined primary and secondary outcome measures and how you assessed these measures. 